\title{
DISFUNCIÓN SEXUAL DE LA MUJER EN EDAD REPRODUCTIVA
}

\author{
Sexual dysfunction in women of reproductive age. \\ *Vilma Mercedes Miranda Baquedano, ${ }^{* *}$ Mercy Violeta Lara Lara
}

\section{RESUMEN}

La disfunción sexual femenina (DSF) es un trastorno que se produce cuando hay cambio en el comportamiento sexual habitual de la mujer; disminuyen o incluso desaparecen los pensamientos y fantasías sexuales, posponen o evitan las relaciones sexuales y existe incapacidad para disfrutar el acto sexual y se presenta entre el $22 \%$ a $43 \%$ de la población en general. Objetivo Conocer la prevalencia de disfunción sexual en la mujer en edad reproductiva de 19 a 35 años en la comunidad del bordo del rio Santa Ana en el I semestre del 2015. Pacientes y Métodos: Estudio de alcance descriptivo, corte transversal, enfoque cuantitativo. La población; 247 mujeres en edad fértil (de 19 a 35 años), muestra de 71 mujeres que consintieron participar. Criterios de exclusión mujeres menores de 19 y mayores de 35 y solteras de 19 a 35 años. La información se obtuvo mediante un instrumento tipo cuestionario con escala licker, procesando los datos en programa EPI-INFO. Resultados Están satisfechas con su vida sexual 36 $(51 \%)$ y $35(49 \%)$ refirieron no estarlo, $21(30 \%)$ tenían satisfacción plena con su vida sexual y $50(70 \%)$ no estaban satisfechas. $35(54 \%)$ de las parejas, no le muestran afecto a la mujer, durante el acto sexual y tampoco colaboran para tener un orgasmo. Conclusiones La prevalencia de la disfunción sexual en la mujer en edad reproductiva es elevada, casi la mitad está sufriendo algún trastorno en las fases de deseo, excitación, dolor o lubricación lo que le impide tener una adecuada respuesta sexual humana.

PALABRAS CLAVE: Conducta sexual, periodo fértil, salud sexual y reproductiva.

\section{ABSTRACT}

Female Sexual Dysfunction (FSD). is a disorder a woman's that occurs when there is a change in sexual behavior. Sexual thoughts and fantasies diminish or even disappear and, postpone or avoid sexual intercourse. In addition, there is an inability to enjoy sex it. Occurs to $22 \%$ to $43 \%$ of the general population. Objective: To know the prevalence of sexual dysfunction in women of reproductive ages in the community of the Santa Ana River during the first half of 2015. Patients and Methods: Cross-sectional descriptive study. The population of 247 women of childbearing age (19 to 35 years), sample of 71 women participating with prior informed consent. Exclusion criteria women under in ages 18 and over 35 . The information was obtained through a Instrument type questionnaire with licker scale, processing the data in the EPI-INFO program. $36(50.7 \%)$ and $35(49.3 \%)$. Results: They were satisfied with their sex life, $36(51 \%)$ and $35(49 \%)$ reported not being present, $21(30 \%)$ had full satisfaction with their sex life and $50(70 \%)$ were not satisfied. 35 (54\%) of the couples do not show affection to the woman during sex and don't collaborate to have an orgasm Conclusions The prevalence of sexual dysfunction in women of reproductive age is significant, almost half are suffering from a disorder in the phases of desire, excitement, pain or lubrication, which prevents them from having an adequate human sexual response.

KEYWORDS: Sexual behavior, fertile period, sexual and reproductive health.

INTRODUCCIÓN. Según la Organización Mundial de la Salud (OMS ,1992.) la disfunción sexual es la dificultad o imposibilidad del individuo de participar en las relaciones sexuales tal como lo desea. La Disfunción Sexual Femenina (DSF) ocurre en un $22 \%$ a $43 \%$ de la población general" (1) La disfunción sexual se manifiesta como una alteración o trastorno en la respuesta sexual humana, más específicamente, en las fases del deseo, excitación, orgasmo, o dolor asociado al coito. El acto sexual va relacionado con la sexualidad y esta se define como las diferentes maneras en que experimentamos y nos expresamos como seres sexuados por naturaleza. ${ }^{(2)}$

La salud sexual y el disfrute de la misma cambia de una cultura a otra, la salud sexual es definida

\footnotetext{
*Profesora de la Carrera de Enfermería de la Escuela Universitaria de Ciencias de la salud (EUCS) de la Universidad Nacional Autónoma de Honduras en el Valle de Sula (UNAH - VS). Licenciada en Enfermería. Especialista en Salud Materno Perinatal. Magister en Gestión Educativa.

${ }^{* *}$ Estudiante de la Carrera de Enfermería de la EUCS-UNAHVS.

Dirigir correspondencia a: vilmamiranda2012@yahoo.es

Recibido: 25 de octubre $2016 \quad$ Aprobado: 01 de mayo 2017
} 
por la Organización Mundial de la Salud como el estado de bienestar físico, emocional, mental y social con relación a la sexualidad; no es la mera ausencia de enfermedad, disfunción o incapacidad. La salud sexual necesita un acercamiento positivo y respetuoso a la sexualidad y a las relaciones sexuales, así como, la posibilidad de obtener experiencias placenteras y seguras, libre de coacción, discriminación y violencia, la salud sexual debe defender, proteger, mantener y respetar los derechos sexuales de las personas. ${ }^{(3)}$

En la sexualidad se han identificado trastornos o disfunciones principalmente en las mujeres, actualmente se ha abierto el campo del tratamiento de estas (también conocidas como disfunciones eróticas). El erotismo es la potencialidad humana de producir y comunicar una forma especial de placer: deseo, excitación y orgasmo. ${ }^{(4)}$ Masters y Jhonson (en su libro Human sexual response) son los primeros que establecen un modelo fisiológico de respuesta sexual humana definiendo cuatro etapas que se expresan de forma diferente en varones y mujeres: excitación, meseta, orgasmo y resolución. ${ }^{(5)}$

A nivel fisiológico, y con independencia del modelo que adoptemos, existe una primera fase (deseo) en la que aumenta la tensión somática, mediada por complejos sistemas hormonales, experienciales y culturales. Aunque las respuestas son muy similares entre hombres y mujeres, las emociones y pensamientos de los seres humanos son muy complejos, por tanto no resulta sorprendente que las respuestas sexuales varíen en forma considerable entre los distintos individuos. ${ }^{(6)}$ Antes de los trabajos de Masters y Johnson, las disfunciones sexuales eran consideradas síntomas de otras enfermedades y se abordaban con una sola óptica, de tal manera que todas las alteraciones sexuales se trataban de la misma forma. Las disfunciones sexuales son una serie de síndromes en los que los procesos eróticos de la respuesta sexual resultan no deseables para el individuo o para el grupo social y que se presentan en forma recurrente y persistente. ${ }^{(7)}$

Algunos autores valoran los siguientes aspectos psíquicos en toda disfunción sexual: ansiedad de actuación, ignorancia y errores sexuales, dificultades en las relaciones, baja autoestima, actitudes negativas en relación con la actividad sexual, creencias religiosas o la educación recibida, circunstancias adversas. ${ }^{(8)}$ Abraham Maslow (en su libro Motivation and Personality), ${ }^{(9)}$ presentó una forma muy sencilla de concebir las necesidades de una persona. La teoría de Maslow plantea que las necesidades inferiores son prioritarias, y por lo tanto, más potente que las necesidades superiores de la jerarquía. Solamente cuando la persona logra satisfacer las necesidades inferiores, entran gradualmente en su conocimiento las necesidades superiores. Al guiar esta investigación en base a la teoría de Maslow se afirma que las necesidades verdaderamente básicas del hombre son la alimentación, respiración, descanso, homeostasis (condición interna estable) y el sexo. Es decir, una vez más se aduce que el hombre es un ser biológicamente sexual, esta necesidad no se refiere únicamente al acto sexual también están asociadas el deseo sexual, el comportamiento maternal, el erotismo entre otras. Lo ideal debería de ser que la sexualidad femenina avance en nuestros días y despierte de todos los tabúes y restricciones que ha sufrido por décadas, una discriminación sexual total que le ha impedido demostrar sus molestias, sensaciones, pensamientos, fantasías y deseos, para llegar a ser una parte integral de la mujer y complementarla en su desarrollo como persona y como pareja. Según varios autores la disfunción sexual femenina puede deberse a una causa física, ser inducida por sustancias o ser de tipo no orgánica, cada tipo de trastorno de la respuesta sexual humana posee diversas causas que la producen. Las disfunciones sexuales no se diagnostican con frecuencia, porque las pacientes sienten vergüenza y no saben cómo hablar sobre estos problemas. La mejor edad reproductiva de una mujer es cerca de los 20 años. La fertilidad disminuye gradualmente a partir de los 30 años, sobre todo después de los 35 años. ${ }^{(10)}$

Las mujeres con trastornos de disfunción sexual no están tomando ninguna medida alternativa para afrontar dicha anormalidades lo que deja como resultado mujeres que no disfrutan su sexualidad plenamente y tienen consecuencias biopsicosociales lo que es respaldado por las aseveraciones de autores que opinan que el grado de sufrimiento y frustración que se deriva de las DSF produce una serie de desajustes psíquicos ${ }^{(11)}$ Las disfunciones sexuales reducen la calidad de vida de muchas mujeres, produciendo con frecuencia un sufrimiento suficiente para diagnosticar un trastorno sexual.

El objetivo del presente estudio, fue conocer la prevalencia de la disfunción sexual de la mujer en edad reproductiva de 19 a 35 años en la comunidad del bordo del rio Santa Ana, durante el I semestre del año 2015. 


\section{PACIENTES Y MÉTODOS}

Se realizó un estudio descriptivo, de corte transversal, en la comunidad del Bordo del Rio Santa Ana sector Pedregal ubicada en el departamento de Cortés, municipio de San Pedro Sula, durante el I semestre del año 2015. Población 271, por muestreo probabilístico aleatorio simple se obtuvieron 151 mujeres, sin embargo la muestra estuvo constituida por 71 mujeres que aceptaron participar bajo consentimiento informado. Se aplicó un instrumento de recolección de datos tipo cuestionario, con escala de licker que constaba de 13 preguntas agrupadas en 2 variables:

1. Disfunción sexual femenina, dentro de la cual se tomaron las dimensiones de trastorno del deseo, excitación, dolor, trastorno del orgasmo.

2. Mujer en edad reproductiva (19 a 35 años), que posee las dimensiones biológica y social.

Los datos se analizaron mediante Epi-Info 7 y el paquete estadístico de Excel 2010.

\section{RESULTADOS}

El estudio se realizó en 71 mujeres, de las que $29(40.8 \%)$ tenían entre 25-30 años. Ver tabla No.1.

Tabla No. 1 Edad de las mujeres

\begin{tabular}{|ccc|}
\hline Edad & $N^{\circ}$ & $\%$ \\
\hline $19-241$ & 7 & 23.9 \\
\hline $25-302$ & 9 & 40.8 \\
\hline $31-352$ & 5 & 35.3 \\
\hline Total7 & 1 & 100 \\
\hline
\end{tabular}

Fuente. Instrumento sobre disfunción sexual. Familiar I

En relación a la satisfacción en su vida sexual 36 $(51 \%)$, estaban satisfechas. Ver Gráfico No.1.

\section{Grafico No 1. Satisfacción sexual de mujer en edad reproductiva}

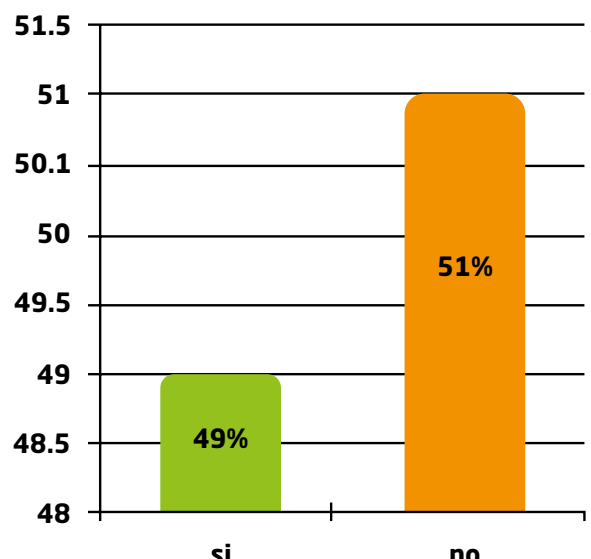

Fuente. Instrumento sobre Disfunción Sexual.
En relación a los juegos eróticos previo al acto sexual, 38 (54\%) si los practicaban. Ver Gráfico No. 2

\section{Gráfico No. 2. Juegos eróticos previo al acto sexual de las mujeres}

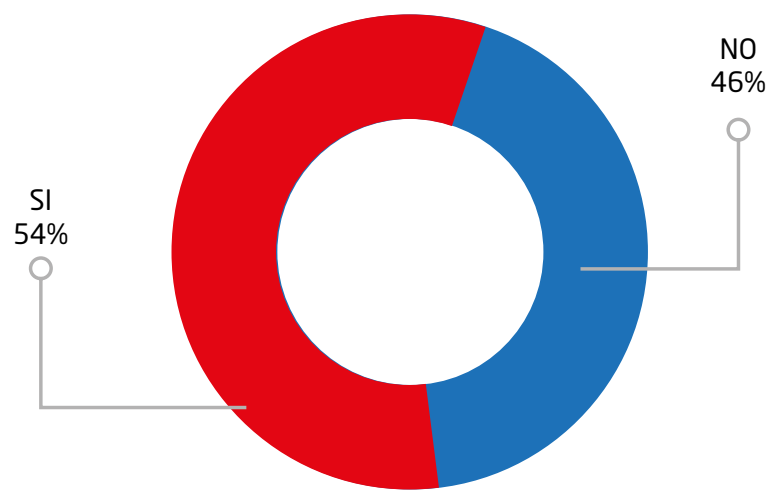

Fuente. Instrumento sobre Disfunción Sexual.

Se encontró que $14(20 \%)$ de las mujeres iniciaban el acto sexual y $80 \%$ no. $54(76 \%)$ tenían interés por las relaciones sexuales y $24 \%$ no tenían interés. $21(30 \%)$ tenían satisfacción plena con su vida sexual y $50(70 \%)$ no estaban satisfechas. Ver Gráfico No. 3

\section{Gráfica No. 3. Actividades e interés de la mujer en la} relación sexual

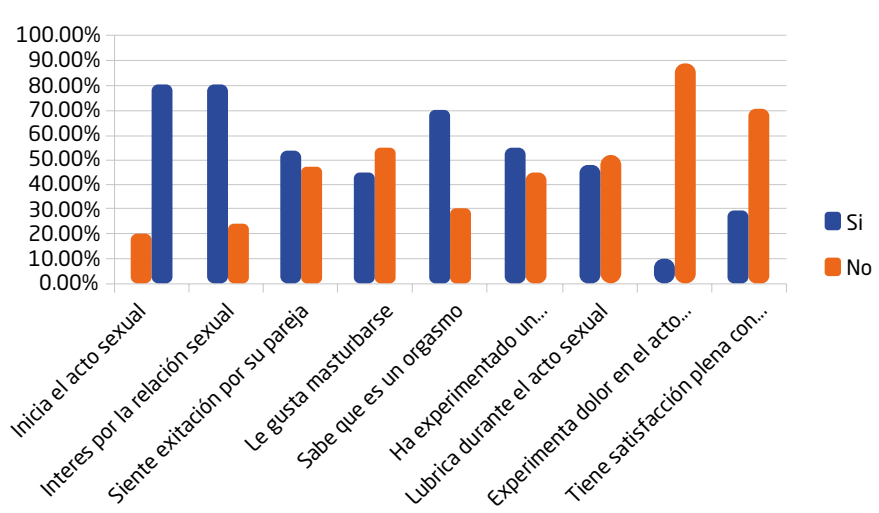

Fuente. Instrumento sobre Disfunción Sexual.

Se encontró que 35(54\%) de las parejas, no le muestran afecto a la mujer, durante el acto sexual y tampoco colaboran para que tengan un orgasmo.

Ver tabla No. 2. 
Tabla No. 2. Interés de la pareja en satisfacer a la mujer

\begin{tabular}{|l|r|r|r|}
\hline \multicolumn{1}{|c|}{ ActividadS } & iN & oT & otal \\
\hline Le muestra afecto en al acto sexual & $33(46 \%)$ & $35(54 \%)$ & $100 \%$ \\
\hline $\begin{array}{l}\text { Colabora para que tenga un } \\
\text { orgasmo }\end{array}$ & $33(46 \%)$ & $35(54 \%)$ & $100 \%$ \\
\hline
\end{tabular}

\section{DISCUSIÓN.}

El trastorno de la respuesta sexual humana es multifactorial; entrelazado a esto está la personalidad de la mujer, factores de represión como la misma religión o la sociedad según la época en que han crecido. La cultura sobre la educación sexual y reproductiva no permite que la mujer disfrute plenamente de su sexualidad. Las DSF se dan más en las clases humildes con bajos niveles de educación (11) y el estudio fue realizado en una comunidad con escasos recursos económicos y con múltiples necesidades básicas insatisfechas por lo cual es difícil justificar un trastorno sin considerar el entorno. La mujer en edad reproductiva investigada oscila en la edad de 19 a 35 años y según la OMS (Organización Mundial de la Salud), cerca del 40 al $50 \%$ de las mujeres adultas tienen al menos una disfunción sexual la cual repercute en la calidad de vida. ${ }^{(12)}$

Un estudio en Valladolid, España, reporta que el 30 a $50 \%$ de las mujeres experimenta disfunción sexual en algún momento de su vida ${ }^{(13)}$ y coincide con las opiniones de autores que explican que la mujer experimenta disminución o ausencia del deseo y del placer que se basa en una impresión subjetiva de insatisfacción de la vida sexual que puede ser reflejo de disfunción sexual. ${ }^{(8)}$ De la población encuestada, al abordarla sobre su satisfacción sexual, unicamente el $51 \%$ estaba satisfecha, datos similares a un estudio en México sobre satisfacción sexual en 100 parejas de casados: el $49 \%$ de los participantes estaba satisfecho sexualmente: $46 \%$ de las mujeres y $52 \%$ de los hombres ${ }^{(14)}$

Dentro de estos porcentajes de insatisfacción sexual la fase de la RSH ( Respuesta sexual Humana) que se verá más perjudicada con esto es la del orgasmo debido a que las mujeres de este estudio manifiestan saber que es un orgasmo $(70 \%)$, pero no experimentarlo (45\%) ni tener ayuda de su pareja para lograrlo, lo cual dificulta más lograr el acto y para que la mujer logre el orgasmo se requiere una buena pareja, buen ritmo y un acto sexual prolongado, ${ }^{(15)}$ contrario a ello, en este estudio $54 \%$ de las parejas no colabora ni muestra afecto durante el acto sexual, tampoco ayudan para que la mujer experimente un orgasmo, lo que pone de manifiesto la importancia de la integración de la pareja por eso es útil que la mujer discuta con el hombre sus temores y fantasías respecto al orgasmo.

Un estudio en Chile sobre la respuesta sexual femenina concluye que si una mujer es incapaz de sentir orgasmos, es frecuente que tampoco disfrute con otros aspectos del juego amoroso y pierda también gran parte de su apetito sexual, (16) lo que concuerda con este estudio en donde más de la mitad de las mujeres no practica juegos eróticos o amorosos.

Se encontró que $45 \%$ de las mujeres de este estudio no se masturba lo que contrasta con un estudio realizado en México en 100 parejas en donde el $65 \%$ de las mujeres nunca se ha masturbado en relación al hombre que solo el $32 \%$ no lo ha hecho ${ }^{(14)}$ y es concordante con la cultura latina represora de la sexualidad femenina en relación al sexo con el cónyuge ${ }^{(17)}$

La ira o la repulsión hacia la pareja sexual o el miedo a la intimidad pueden causar dolor durante las relaciones sexuales. ${ }^{(18)}$ un estudio sobre dispareunia en España, encontró muy pocas causas orgánicas que la expliquen, lo que supone que hay un gran componente psicológico como los originados por los conflictos de pareja. El miedo al embarazo en la mujer, que el hombre sea infiel y desconsiderado con ella, que busque la prostitución y no quiera practicarse la vasectomía, fueron los conflictos que más afloraron. ${ }^{(19)}$ En este estudio se encontró que únicamente el $11 \%$ de mujeres presentaban dolor durante el acto sexual.

Conclusión: La prevalencia de disfunción sexual femenina es un poco más del $50 \%$ y presentan, entre los aspectos más relevantes la limitada integración del hombre al desenvolvimiento de la sexualidad de la mujer, un tema muy arraigado a la cultura hondureña y de países en vías de desarrollo donde se manda al hombre a experimentar plenamente su sexualidad convirtiendo a la mujer en un ser sumiso y dominado.

Recomendación: Profundizar en las causas que originan este trastorno para conocer si son de etiología orgánica o no orgánica elaborando un estudio de alcance analítico y así poder ofrecer respuesta a hombres y mujeres en su salud sexual. 


\section{REFERENCIAS BIBLIOGRAFICAS}

1. Bechara A, Casabe A, Bertolino V. Disfunción sexual femenina. Rev. Arg de Urol. [En Internet] 2009 [Acceso 22 de marzo del 2015];67(4):187-202 Disponible en: https://studylib.es/doc/7445955/disfuncion-sexual-femenina.

2. Rathus SA, Nevid, JS, Fichner-Rathus L. Sexualidad Humana. $6^{a}$ ed. Madrid: PEARSON EDUCACION; 2005

3. Organización Panamericana de la Salud. Promoción de la salud sexual: recomendaciones para la acción. [EnInternet]. Guatemala:OPS;2000[Acceso 22 marzo del 2015]. Disponible en: http://www1. paho.org/spanish/hcp/hca/salud_sexual.pdf

4. Hablemos de la sexualidad. [sede web] s.l.: Study.lib.es. 2016. [Actualizado en 2016 acceso el 22 de febrero del 2016] Erotismo, respuesta sexual humana y disfunciones eróticas: Módulo 5 Disponible en: http://www. bunam.unam.mx/Sexualidad/SitioSexualidad/ historias/libreropaola/respuestasexual.pdf

5. Gutiérrez Teira B. La respuesta sexual humana. AMF [En Internet] 2010 [Acceso el 21 de abril 2016];6(10):543-546. Disponible en: http://amf-semfyc.com/upload_articles_pdf/La_respuesta_sexual_humana.pdf

6. Reeder S, Martin L, Koniak D. Enfermería Materno Infantil. $17^{a}$ ed. México: Interamericana; 1995

7. Rubio Aurioles E, Díaz Martínez J. Las disfunciones sexuales: antología de la Sexualidad Humana. [En internet] México: CONAPO 1994 [Acceso el 12 de mayo del 2015] Disponible en : http://www.facmed.unam.mx/deptos/ familiar/compendio/Primero/I_SM_260-303.pdf

8. Cañones Garzon PJ, Aizpiri Díaz J, Barbado Alonso JA, Fernández Camacho A, Goncalves Estella F, Rodríguez Sendín JJ, et al. Disfunciones sexuales de origen no orgánico. Medicina General [En internet] 2002. [Acceso 08 de abril del 2015]; 49:916-922. Disponible en: https://studylib.es/doc/8528194/916-disfunciones-sexuales-de-origen-no-org \% C3\%A1nico

9. Gestionopolis. Jerarquía de las necesidades de Abraham Maslow. [sede web]. S.I.:Webprofit Ltda; 2002 [Actualizado 2017, Acceso el 1 de abril del 2015]. Disponible en http://www.gestiopolis.com/jerarquia-de-las-necesidades-de-abraham-maslow/

10. American Society for Reproductive Medicine. Edad y fertilidad: guía para pacientes. [ En Internet]. Alabama: American Society for Reproductive Medicine; 2013. [Acceso 30 de abril del 2015]. Disponible en: http://www.reproduc- tivefacts.org/globalassets/rf/newsandpublications/bookletsfact-sheets/spanish-fact-sheetsand-info-booklets/edad_y_fertilidad-spanish.pdf

11. Pintado Vázquez S. Disfunción sexual femenina en la menopausia: impacto sobre la calidad de vida. Rev Int Androl [ En internet] 2007. [ Acceso de 11 de junio 2016]; 5(3):28996. Disponible en: http://apps.elsevier.es/ watermark/ctl_servlet? $f=10 \& p i d e n t$ articuIo $=13110299 \&$ pident usuario $=0 \&$ pcōntacti$\mathrm{d}=$ \&pident_revista $=2 \overline{6} 2 \& \mathrm{ty}=71 \&$ accion $=$ L\&origen $=$ zona delectura \&web $=w w w$. elsevier. es\&lan=es\&fichero=262v5n3a13110299pdf001.pdf

12. Organización Panamericana de la Salud. Salud sexual para el milenio: declaración y documento técnico. [EnInternet]. Washington:OPS;2009. [Acceso 15 de ene. 2013). Disponible en: http://www. asumen.org.ar/pdf/Salud_Sexual_Milenio.pdf

13. Huallpa Chirinos AM, Juro NS. Una exploración de la disfunción sexual femenina no orgánica en adultas tempranas atendidas en un establecimiento del Ministerio de Salud de la Lima Metropolitana 2013. [Tesis Pregrado]. Perú: Universidad Nacional de San Marcos; 2013.

14. BridgesSK, LeaseSH,EllisonCR.Predictingsexual satisfaction in women: implications for counselor education and training. JCD 2004; 82(1):158-166.

15. Navarro Arias R. Psicoenergetica, método de psiciterapia corporal para integrar las emociones y el cuerpo. Mexico: Pax; 2007

16. Kamei J. Análisis de la respuesta sexual femenina y grado de satisfacción sexual en parejas de hombres tratados de una disfunción eréctil. Rev chil Urol. 2005; 70(4):236-239.

17. PaternostroS. IntheLand ofGodandMan:Confronting our Sexual Culture. NewYork: Hardcover; 1998

18. Gori R. Ginecología de Gori. $2^{\mathrm{a}}$. Ed. Buenos Aires: Ateneo; 2005

19. López-Olmos J. Dispareunia: investigación de causa física y de causa infecciosa crónica: estudio prospectivo de 4 años. Clín Invest Gin Obste [en internet] 2008 [Acceso 04 de sept. 2016]; 35(5):1529. Disponible en: http://www.sciencedirect. com/science/article/pii/S0210573X08730685 